\title{
Influência do uso do Wii Fit sobre o equilíbrio estático e a marcha na doença de Parkinson
}

\section{Influence of the use of Wii Fit on static balance and gait in Parkinson disease}

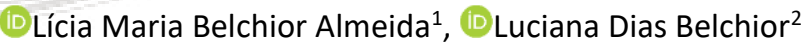

1 Universidade Federal do Ceará - UFC

2 Universidade de Fortaleza - UNIFOR

\section{Correspondência}

Lícia Maria Belchior Almeida

E-mail: liciamba@hotmail.com

Submetido: 25 Junho 2020

Aceito: 14 Agosto 2020

Como citar

Almeida LMB, Belchior LD. Influência do uso do Wii Fit sobre o equilíbrio estático e a marcha na doença de Parkinson. Acta Fisiatr. 2020;27(2):107-112.

DOI: 10.11606/issn.2317-0190.v27i2a171459

\section{cc) (1) ()}

\section{RESUMO}

A Doença de Parkinson (DP) caracteriza-se, especialmente, por bradicinesia, instabilidade postural, reflexos reduzidos e alteração de marcha, aumentando o risco de quedas. Objetivo: Avaliar a influência de exercícios protocolados sobre equilíbrio estático e marcha com Wii Fit na DP. Métodos: Trata-se de artigo original que analisou alterações estáticas e marcha em dois pacientes com Parkinson, submetidos ao Nintendo Wii e avaliados antes e após o protocolo. A avaliação deu-se mediante análise do equilíbrio estático e da marcha, no início e ao final de 6 semanas após o protocolo de três jogos: Penguin Slide, Plataforms e Balance Bubble, 2 vezes semanais, abordando a mesma sequência. A pesquisa foi realizada no Laboratório de Análise do Movimento Humano, vinculado à Universidade de Fortaleza, de fevereiro a setembro de 2015. Resultados: Os dados baropodométricos, referentes à variação da superfície, mostraram valor menor após intervenção. A variável velocidade média aumentou após atividade. As distâncias entre 2 ㅇ e 3 ㅇ passo e entre 3 e 4 ㅇ passo também aumentaram, após intervenção, exceto esta última para o primeiro paciente. Com o protocolo de Nitendo Wii Fit, participantes relataram maior agilidade e segurança nas passadas, podendo ser comprovado pelo aumento da velocidade média dessas. Os pacientes evidenciaram uma melhora no equilíbrio estático e na marcha. Conclusão: Os exercícios protocolados em realidade virtual nos pacientes com DP melhoraram o desempenho do equilíbrio estático e da marcha, o que tende a favorecer a estabilidade ortostática e a deambulação.

Palavras-chave: Doença de Parkinson, Jogos de Vídeo, Exercício de Simulação, Equilíbrio Postural, Análise da Marcha

\section{ABSTRACT}

Objective: This study aimed to compare the painful pressure threshold in the pelvic floor of elderly women with and without fibromyalgia. Methods: A double blind study was performed with 28 elderly women aged 60 to 75 years, divided into two groups, with medical diagnosis of fibromyalgia (FG) and without fibromyalgia (GWF). The evaluation was performed in a single meeting, on an individual basis, in which data on the gynecological, obstetrical, and medical history and information regarding the pelvic floor were obtained by previously trained volunteers. The evaluation of pressure pain threshold was performed with the use of an algometer by one of the researchers. Two-tailed Student t test, Mann-Whitney $U$ Test and Chi-square tests were used. All tests were performed with a significance level of $5 \%$. Results: The pain pressure threshold showed that FG presented lower values for pain perception in relation to the GWF ( $p<0.001$ for the left side) and ( $p<0.001$ for the right side). Conclusion: FG had lower pressure pain threshold, which may be related to the pathology, which causes generalized pain and central sensitization.

Keywords: Parkinson Disease, Video Games, Simulation Exercise, Postural Balance, Gait Analysis 


\section{INTRODUÇÃO}

Doenças crônicas e progressivas caracterizam-se pela ausência de intervalos ou períodos de alívio dos sintomas, desenvolvendo espontaneamente efeitos progressivos e severos. Dentre tais doenças incapacitantes, encontra-se a Doença de Parkinson (DP), doença crônica e neurodegenerativa ligada aos gânglios da base, com incidência em população acima de 65 anos, sendo a segunda doença neurodegenerativa mais comum, multifatorial, envolvendo aspectos genéticos e ambientais. ${ }^{1}$

O envelhecimento faz parte da patogenia da afecção e o acúmulo de determinados materiais biológicos ou o tempo de exposição às toxinas podem estar envolvidos na mudança genética provocada pelo envelhecimento. ${ }^{2} \mathrm{~A}$ pessoa com tal diagnóstico até a morte pode chegar a 15 anos e a taxa de mortalidade varia entre $1,5 \%$ a $2 \% .^{3}$

A DP, caracterizada como doença progressiva que atinge estruturas do sistema nervoso central, promove incapacidade funcional dos acometidos, pois há comprometimento progressivo dos movimentos. Seu diagnóstico é estabelecido a partir dos sintomas clínicos, principalmente rigidez muscular, tremor de repouso, bradicinesia e alteração postural. ${ }^{4}$

$\mathrm{O}$ tremor é o sintoma mais frequente, sendo rítmico e lento, atingindo principalmente as extremidades ao repouso. Com a progressão, a bradicinesia acentua-se, podendo resultar em imobilidade completa. A rigidez muscular promove resistência aos músculos, tornando-os mais tensos e contraídos deixando o indivíduo rígido e com pouca mobilidade. ${ }^{5}$

A instabilidade postural dos pacientes com DP resulta em uma posição encurvada, prejudicando a marcha. Os reflexos posturais, inclusive o equilíbrio, estão todos diminuídos. Com isso, eleva-se, ainda mais, o risco de quedas e lesões frequentes como consequência. Essa falha postural provoca o deslocamento do centro de gravidade para frente (propulsão) ou para trás (retropulsão), ocasionando a marcha de passos curtos e lentos, mediante o estreitamento da base de apoio. ${ }^{2}$

Sintomas depressivos, distúrbios cognitivos, alterações do sono, dificuldades de fala, sialorréia, dificuldades respiratórias, déficit para deglutição, disfunções urinárias, dores musculares e sensações térmicas anormais constituem sintomas não motores. $^{5}$

Devido a sintomatologia e a debilidade funcional de indivíduos com DP, a necessidade de intervenção voltada para melhora na qualidade de vida e manutenção funcional desses é alta, e os jogos de realidade virtual (RV) vêm tomando espaço nessa linha terapêutica. ${ }^{6,7}$

O Nintendo Wii consiste em jogos com plataforma que capta e mede dados referentes ao peso, centro gravitacional e pressão dos pés, e com esses dados aliados ao jogo "Wii Fit", é possível realizar trabalhos de coordenação, percepção espacial, posturais e de equilíbrio. ${ }^{8}$

As variedades de jogos interativos do Wii Fit incrementam aptidão motora e sintomatologias psíquicas de isolamento social, pois estimulam os participantes a alcançarem pontuações mais altas através da competição de pontos no console. $^{9}$

O presente estudo justifica-se pela tendência de envelhecimento da população nacional e pelo consequente aumento dos casos da Doença de Parkinson, o que explicita a necessidade de novas técnicas adjuvantes os quais possam contribuir com o aumento da qualidade de vida desses idosos e com a redução de gastos públicos com incapacidades.

\section{OBJETIVO}

O presente estudo tem como objetivo avaliar a influência de exercícios protocolados sobre equilíbrio estático e marcha com Wii Fit em pacientes com a Doença de Parkinson.

\section{MÉTODOS}

Estudo de caso que analisou as alterações estáticas e a marcha em dois pacientes do ambulatório de neurologia do Núcleo de Atenção Médica Integrada (NAMI), que se encontravam em mesmo esquema medicamentoso para tratamento de Parkinson (medicamento dopaminérgico levodopa ou agonista da dopamina, posologia: 70 a $100 \mathrm{mg}$, duas vezes ao dia), submetidos ao Nintendo Wii comparados consigo mesmos, antes e após protocolo.

A avaliação foi realizada no Laboratório de Análise do Movimento Humano, vinculado à Universidade de Fortaleza (UNIFOR), e protocolo administrado no NAMI, de fevereiro a setembro de 2015.

Após concordância para participar da pesquisa, os participantes assinaram Termo de Consentimento Livre e Esclarecido.

O presente estudo foi realizado após a aprovação do Comitê de Ética da UNIFOR, sob no do parecer: 969.078. Os pacientes foram submetidos à avaliação do equilíbrio estático e da marcha, antes e após intervenção mediante baropodometria, depois à aplicação do protocolo mediante três jogos do Nintendo Wii Fit ${ }^{\circledR}$ : Penguin Slide, Plataforms e Balance Bubble, por 6 semanas, 2 vezes semanais, totalizando 12 intervenções, 3 exercícios por 15 minutos, na mesma sequência. Inicialmente, realizou-se o protocolo apenas para fins de aprendizado.

Para tal estudo, os indivíduos encontravam-se com DP estágio II e III da escala de estágio de incapacidade de Hoehn e Yahr, entre 60-80 anos e com o mesmo esquema medicamentoso dopaminérgico. ${ }^{10} \mathrm{Na}$ ficha de avaliação clínica constavam dados como idade, sexo, altura, peso, tempo de patologia estabelecida e de atividade física.

A análise do equilíbrio estático e da marcha dos participantes constou de avaliação realizada com baropodômetro eletrônico de dois metros de comprimento (FootWork Pro, AM CUBE, França), com frequência de amostragem de $200 \mathrm{~Hz}$, adaptado à pista de marcha com comprimento total de oito metros, permitindo aceleração e desaceleração da marcha nos três metros iniciais e finais. As análises deram-se com auxílio de software FootWork Pro versão 3.7.0.1 (IST Informatique - Intelligence Service et Tecnique, França).

Antes do teste estático, cada paciente foi solicitado a permanecer vinte segundos em posição ortostática no baropodômetro, braços ao longo do corpo e olhar fixo. Este procedimento não foi repetido na análise da marcha, porém, foram capturados dados a partir da segunda volta do paciente na esteira, permitindo adaptação.

Para a avaliação da marcha, um por vez, solicitou-se deambular por percurso de oito metros de comprimento, 
sendo capturados dados nos dois metros intermediários, correspondente a área útil do baropodômetro.

Ambos os pacientes passaram por adaptação ao equipamento previamente à coleta de dados, visando minimizar alterações e não habituação ao meio. Os testes foram realizados na fase off do ciclo de medicação da dopamina, uma vez que a interrupção do uso da medicação pelo período de 12 horas não consiste em um risco evidente para os doentes, pois os efeitos de longa duração permanecem. Tal cenário vêm sendo bastante utilizado em pesquisas que visam à avaliação do indivíduo com DP. ${ }^{11}$

O contato com a sola do pé, na posição ortostática ou dinâmica, fornece dados qualitativos sobre o formato do pé e quantitativos em relação à carga e à pressão da impressão plantar, sobre deslocamento do centro de pressão de cada indivíduo, velocidade da marcha $(\mathrm{mm} / \mathrm{s})$, distância da passada (cm) e cadência (passada/min).

Para a análise estática, os pacientes foram orientados a ficar sobre a plataforma, descalços, em posição ortostática, por vinte segundos, com olhos abertos e braços ao longo do corpo, para análise de valores da superfície plantar do pé direito e esquerdo (kpa), divisão de massa anti pé direito e esquerdo (\%), divisão de massa retro pé direito e esquerdo (\%), sagital frente de pé direito e esquerdo (\%), centro gravitacional sagital volta de pé direito e esquerdo (\%), pressão média do pé direito e esquerdo, pressão média do pé direito e esquerdo (kpa), pressão máxima do pé direito e esquerdo (kpa).

Para o protocolo de tratamento com RV, fora utilizado jogos de vídeo da marca Nintendo ${ }^{\circledR}$, denominado Wii, protocolo Wii Fit. Este jogo possui acessório, Balance Board, responsável pela interface entre máquina e jogador.

Houve comparação entre antes e depois da realização dos exercícios pela baropodometria. Selecionou-se três jogos:

- Penguin Slide: personagem principal é um pinguim que fica sobre iceberg; objetiva contato com número máximo de peixes que saltam da água, deslocando-se de uma extremidade a outra sem cair na água, levando à maior pontuação.

- Plataforms: jogador controla a plataforma, em que uma bola ou mais rolam por esta; objetiva colocar as bolas em buracos nas plataformas com diferentes movimentos de pés para que haja diferentes pontos de pressão na balance board, que se inclina segundo o movimento dos pés do jogador.

- Balance Bubble: personagem principal é uma menina que permanece dentro de uma bola de sabão; objetiva realizar percurso pré-determinado em menor tempo, sem que a bola estoure encostando-se à margem do rio; para fazê-lo, o personagem se desloca para frente, para trás e para as laterais, na superfície de um rio.

Os jogos descritos visam aos ajustes posturais anteroposteriores e látero-laterais e coordenação de membros inferiores, assim como controle de equilíbrio e coordenação, os quais influenciam diretamente no desempenho da marcha. ${ }^{12,13}$

Os dados obtidos foram analisados descritivamente, pelo programa Excell 2010.

\section{RESULTADOS}

Trata-se de estudo de caso de dois pacientes, gênero masculino, com diagnóstico clínico de DP, média de 4 anos.
Para a obtenção dos dados sociodemográficos, os pacientes responderam a ficha de avaliação clínica.

\section{Análise Estática da Marcha}

Paciente 1: M.N.O., masculino, 78 anos, 1,60m, 70kg, tempo de patologia estabelecida com mais de 3 anos, uso de medicação ao Parkinson, sedentário.

Para a variável superfície plantar do pé direito, foi de $100,9 \mathrm{~cm}^{2}$ para $95,1 \mathrm{~cm}^{2}$. Quanto à superfície plantar do pé esquerdo, de $111,9 \mathrm{~cm}^{2}$ para $105,6 \mathrm{~cm}^{2}$.

$\mathrm{O}$ antepé direito apresentou divisão de massa de $68 \%$ no início e $49 \%$ após intervenção. Já o antepé esquerdo obteve valores pré-treino de $59 \%$ e, após, de $46 \%$. O retropé direito apresentou divisão de massa de $32 \%$, para $51 \%$. Já o retropé esquerdo obteve valores pré-treino de $41 \%$, chegando a $56 \%$.

Quanto à oscilação ântero-posterior do pé direito, passou de $53 \%$ para $49 \%$. Valores semelhantes no pé esquerdo. $\mathrm{Na}$ oscilação látero-lateral do pé direito, o valor passou de $46 \%$ para $51 \%$. Já no esquerdo de $47 \%$ passando para $51 \%$.

A pressão média do pé direito obteve valores iniciais de 18,2kpa para 20,8kpa. Já a do pé esquerdo foi de 23,3kpa para 19,51kpa. Quando pesquisada a pressão máxima do pé direito, os números iniciais obtidos foram de $36,8 \mathrm{kpa}$ e os finais de $70,5 \mathrm{kpa}$. Já a pressão máxima do pé esquerdo apresentou 67,8kpa passando para 59,5kpa (Tabela 1 ).

Paciente 2: F.A.P., masculino, 70 anos, $1,60 \mathrm{~m}, 78 \mathrm{~kg}$, com tempo de patologia estabelecida com mais de 4 anos, uso de medicação ao Parkinson, sedentário.

Para a variável superfície plantar do pé direito, os valores passaram de $98,6 \mathrm{~cm}^{2}$ para $100,9 \mathrm{~cm}^{2}$. Quanto à superfície plantar do pé esquerdo, os valores foram de $102,1 \mathrm{~cm}^{2}$ para $99,76 \mathrm{~cm}^{2}$.

O antepé direito apresentou divisão de massa de $36 \%$ no início e $49 \%$ depois. Já o esquerdo obteve valores pré-treino de $50 \%$ e, após, de $41 \%$. O retropé direito apresentou divisão de massa de $64 \%$, passando para $51 \%$. Já o retropé esquerdo obteve valores pré-treino de $50 \%$, chegando a $59 \%$.

Em relação à oscilação ântero-posterior do pé direito, passou de $49 \%$ para $50 \%$. Os valores no pé esquerdo foram semelhantes. Na oscilação látero-lateral do pé direito, o valor passou de $50 \%$ para $41 \%$. Já no esquerdo, $51 \%$ passando para $50 \%$.

A pressão média do pé direito obteve valores iniciais de $25,9 \mathrm{kpa}$ para $21,3 \mathrm{kpa}$. Já no pé esquerdo foi de $28,9 \mathrm{kpa}$ para 33.3kpa. Quando pesquisada a pressão máxima do pé direito, inicialmente foi de $94,1 \mathrm{kpa}$ para $72,6 \mathrm{kpa}$. Já a do pé esquerdo apresentou, inicialmente, $100,8 \mathrm{kpa}$ passando para $145,5 \mathrm{kpa}$ (Tabela 1).

\section{Análise Dinâmica da Marcha}

Paciente 1: M.N.O., masculino, 78 anos, 1,60m, 70kg, com tempo de patologia estabelecida com mais de 3 anos, uso de medicação ao Parkinson, sedentário. A distância entre o segundo e o terceiro passos antes da intervenção com o Nintendo $W i^{\oplus}{ }^{\oplus}$, foi de $10,66 \mathrm{~cm}$, já no pós-tratamento, foi de $12,19 \mathrm{~cm}$. Já a distância entre o terceiro e o quarto passos foi de $13,71 \mathrm{~cm}$ para $9,90 \mathrm{~cm}$. 
Quanto à distância da passada direita, considerando-se o segundo e o quarto passo, inicialmente foi de $70,7 \mathrm{~cm}$ e, após submissão reduziu para $61,7 \mathrm{~cm}$. A mesma variável, do lado esquerdo, teve valores pré-treino de $67,8 \mathrm{~cm}$, e, pós, de $62,5 \mathrm{~cm}$.

$A$ velocidade média à direita, evidenciou valores numéricos de $359,5 \mathrm{~mm} / \mathrm{s}$ para $489,2 \mathrm{~cm}$ após intervenção. A mesma variável, do lado esquerdo, teve valores pré-treino de 239,7 $\mathrm{mm} / \mathrm{s}$, e, após, de 308,2 mm/s (Tabela 2).

Paciente 2: F.A.P., masculino, 70 anos, $1,60 \mathrm{~m}, 78 \mathrm{~kg}$, com tempo de patologia estabelecida com mais de 4 anos, uso de medicação ao Parkinson, sedentário.
A distância entre o segundo e o terceiro passos antes da intervenção com o Nintendo Wii foi de $10,5 \mathrm{~cm}$, já no pós foi de $13,0 \mathrm{~cm}$. Já a distância entre o terceiro e o quarto passos, prévia ao protocolo, mostrou valor de $14,6 \mathrm{~cm}$, e, no final de 6 semanas decresceu para $11,4 \mathrm{~cm}$.

Quanto à distância da passada direita, considerando-se o segundo e o quarto passo, inicialmente foi de $51,8 \mathrm{~cm}$ e reduziu para $67,8 \mathrm{~cm}$. A mesma variável, do lado esquerdo, foi de $52,6 \mathrm{~cm}$ para $63,2 \mathrm{~cm}$. A velocidade média à direita, antes do protocolo, foi de $250,4 \mathrm{~mm} / \mathrm{s}$ para $265,2 \mathrm{~mm} / \mathrm{s}$. Essa variável, do lado esquerdo, foi de $178,5 \mathrm{~mm} / \mathrm{s}$ para $240,4 \mathrm{~mm} / \mathrm{s}$ (Tabela 2).

Tabela 1. Análise estática da marcha

\begin{tabular}{|c|c|c|c|c|c|c|c|}
\hline $\begin{array}{l}\text { Paciente } 1 \\
\text { Direita } \\
\end{array}$ & $\begin{array}{l}\text { Superfície } \\
\text { Plantar D }\end{array}$ & $\begin{array}{c}\text { Divisão de Massa } \\
\text { Antepé D }\end{array}$ & $\begin{array}{c}\text { Divisão de Massa } \\
\text { Retropé D }\end{array}$ & $\begin{array}{c}\text { Oscilação } \\
\text { Ântero-Posterior D }\end{array}$ & $\begin{array}{c}\text { Ossilação } \\
\text { Látero-Lateral D }\end{array}$ & $\begin{array}{l}\text { Pressão } \\
\text { Média D } \\
\end{array}$ & $\begin{array}{l}\text { Pressão } \\
\text { Máxima D }\end{array}$ \\
\hline Inicial & $100,9 \mathrm{~cm}^{2}$ & $68 \%$ & $32 \%$ & $53 \%$ & $54 \%$ & $18,2 \mathrm{kpa}$ & 36,8kpa \\
\hline Final & $95,1 \mathrm{~cm}^{2}$ & $49 \%$ & $51 \%$ & $49 \%$ & $49 \%$ & 20,8kpa & $70,5 \mathrm{kpa}$ \\
\hline $\begin{array}{l}\text { Paciente } 1 \\
\text { Esquerda }\end{array}$ & $\begin{array}{l}\text { Superfície } \\
\text { Plantar E }\end{array}$ & $\begin{array}{c}\text { Divisão de Massa } \\
\text { Antepé E }\end{array}$ & $\begin{array}{c}\text { Divisão de Massa } \\
\text { Retropé E }\end{array}$ & $\begin{array}{c}\text { Oscilação } \\
\text { Ântero-Posterior E }\end{array}$ & $\begin{array}{c}\text { Ossilação } \\
\text { Látero-Lateral E }\end{array}$ & $\begin{array}{l}\text { Pressão } \\
\text { Média E }\end{array}$ & $\begin{array}{l}\text { Pressão } \\
\text { Máxima E }\end{array}$ \\
\hline Inicial & $111,9 \mathrm{~cm}^{2}$ & $59 \%$ & $41 \%$ & $53 \%$ & $47 \%$ & $23,3 \mathrm{kpa}$ & 67,8kpa \\
\hline Final & $105,6 \mathrm{~cm}^{2}$ & $46 \%$ & $56 \%$ & $49 \%$ & $51 \%$ & 19,51kpa & 59,5kpa \\
\hline $\begin{array}{l}\text { Paciente } 2 \\
\text { Direita }\end{array}$ & $\begin{array}{l}\text { Superfície } \\
\text { Plantar D }\end{array}$ & $\begin{array}{c}\text { Divisão de Massa } \\
\text { Antepé } D\end{array}$ & $\begin{array}{c}\text { Divisão de Massa } \\
\text { Retropé D }\end{array}$ & $\begin{array}{c}\text { Oscilação } \\
\text { Ântero-Posterior D }\end{array}$ & $\begin{array}{c}\text { Ossilação } \\
\text { Látero-Lateral D }\end{array}$ & $\begin{array}{l}\text { Pressão } \\
\text { Média D }\end{array}$ & $\begin{array}{l}\text { Pressão } \\
\text { Máxima D }\end{array}$ \\
\hline Inicial & $98,6 \mathrm{~cm}^{2}$ & $36 \%$ & $64 \%$ & $49 \%$ & $50 \%$ & $25,9 \mathrm{kpa}$ & $94,0 \mathrm{kpa}$ \\
\hline Final & $100,92 \mathrm{~cm}^{2}$ & $49 \%$ & $51 \%$ & $50 \%$ & $49 \%$ & $21,2 \mathrm{kpa}$ & $72,6 \mathrm{kpa}$ \\
\hline $\begin{array}{l}\text { Paciente } 2 \\
\text { Esquerda }\end{array}$ & $\begin{array}{l}\text { Superfície } \\
\text { Plantar E }\end{array}$ & $\begin{array}{c}\text { Divisão de Massa } \\
\text { Antepé E }\end{array}$ & $\begin{array}{c}\text { Divisão de Massa } \\
\text { Retropé E }\end{array}$ & $\begin{array}{c}\text { Oscilação } \\
\text { Ântero-Posterior E }\end{array}$ & $\begin{array}{c}\text { Ossilação } \\
\text { Látero-Lateral E }\end{array}$ & $\begin{array}{l}\text { Pressão } \\
\text { Média E }\end{array}$ & $\begin{array}{l}\text { Pressão } \\
\text { Máxima E }\end{array}$ \\
\hline Inicial & $102,0 \mathrm{~cm}^{2}$ & $50 \%$ & $50 \%$ & $49 \%$ & $50 \%$ & $28,9 \mathrm{kpa}$ & $100,8 \mathrm{kpa}$ \\
\hline Final & $99,7 \mathrm{~cm}^{2}$ & $41 \%$ & $59 \%$ & $50 \%$ & $49 \%$ & 33,3kpa & $145,4 \mathrm{kpa}$ \\
\hline
\end{tabular}

D: direita; E: esquerda; cm2: centímetros quadrados; kpa: quilopascal

Tabela 2. Análise dinâmica da marcha

\begin{tabular}{|c|c|c|c|c|}
\hline Paciente 1 - Direita & Distância Passos 2-3 & Distância Passos 3-4 & Distância da Passada D & Velocidade Média D \\
\hline Inicial & $10,6 \mathrm{~cm}$ & $13,7 \mathrm{~cm}$ & $70,0 \mathrm{~cm}$ & $359,5 \mathrm{~mm} / \mathrm{s}$ \\
\hline Final & $12,1 \mathrm{~cm}$ & $9,9 \mathrm{~cm}$ & $61,6 \mathrm{~cm}$ & $489,1 \mathrm{~mm} / \mathrm{s}$ \\
\hline Paciente 1 - Esquerda & Distância Passos 2-3 & Distância Passos 3-4 & Distância da Passada E & Velocidade Média E \\
\hline Inicial & $10,6 \mathrm{~cm}$ & $13,7 \mathrm{~cm}$ & $67,7 \mathrm{~cm}$ & $239,7 \mathrm{~mm} / \mathrm{s}$ \\
\hline Final & $12,1 \mathrm{~cm}$ & $9,9 \mathrm{~cm}$ & $62,4 \mathrm{~cm}$ & $308,1 \mathrm{~mm} / \mathrm{s}$ \\
\hline Paciente 2 - Direita & Distância Passos 2-3 & Distância Passos 3-4 & Distância da Passada D & Velocidade Média D \\
\hline Inicial & $1,52 \mathrm{~cm}$ & $4,7 \mathrm{~cm}$ & $51,7 \mathrm{~cm}$ & $250,3 \mathrm{~mm} / \mathrm{s}$ \\
\hline Final & $12,9 \mathrm{~cm}$ & $11,4 \mathrm{~cm}$ & $67,7 \mathrm{~cm}$ & $265,2 \mathrm{~mm} / \mathrm{s}$ \\
\hline Paciente 2 - Esquerda & Distância Passos 2-3 & Distância Passos 3-4 & Distância da Passada E & Velocidade Média E \\
\hline Inicial & $1,52 \mathrm{~cm}$ & $4,7 \mathrm{~cm}$ & $52,5 \mathrm{~cm}$ & $178,4 \mathrm{~mm} / \mathrm{s}$ \\
\hline Final & $12,9 \mathrm{~cm}$ & $11,4 \mathrm{~cm}$ & $63,2 \mathrm{~cm}$ & $240,4 \mathrm{~mm} / \mathrm{s}$ \\
\hline
\end{tabular}

D: direita; E: esquerda; cm: centímetros; $\mathrm{mm} / \mathrm{s}$ : milímetros por segundo

\section{DISCUSSÃO}

Este trabalho verificou a influência de exercícios protocolados com Wii Fit ${ }^{\oplus}$ sobre equilíbrio estático e marcha em pacientes com DP. Nenhum estudo relatou impacto negativo dessa ferramenta treinando-se para qualquer medida de capacidade de equilíbrio, e a maioria tem indicado pelo menos alguma evidência quantitativa ou anedótico de melhoria. ${ }^{8} \mathrm{~A}$ avaliação deu-se através da análise do equilíbrio estático e da marcha, no início e ao final de 6 semanas, após protocolo de três jogos: Penguin Slide, Plataforms e Balance
Bubble, 2 vezes semanais, totalizando 12 intervenções, 3 exercícios de 15 minutos, sempre na mesma sequência. ${ }^{14}$ Essa tecnologia tem sido implantada no tratamento de diversas patologias neurológicas como a paralisia cerebral, as doenças degenerativas do sistema nervoso e a DP. ${ }^{15}$

A relação inversa entre o risco de DP e exercício independe de intervalos etários específicos. ${ }^{16} 0$ presente estudo identificou que, após período de adaptação, não foram apresentadas dificuldades em executar atividades propostas, 
como também relataram melhora na execução das atividades cotidianas.

Ainda que padrão, a intervenção medicamentosa está associada ao desenvolvimento de complicações motoras típicas. Exercícios físicos também podem incitar o manejo do movimento e retardar o avanço da doença, ${ }^{17}$ como aqui evidenciado, em que os participantes faziam uso da medicação padrão ouro para DP e apresentaram maior destreza ao realizar simples atividades. O Nintendo $\mathrm{Wii}^{\circledR}$ também pode ser usado para controle do autodomínio, ${ }^{18}$ pois o paciente com Parkinson apresenta déficit de equilíbrio, tornando-se suscetível a quedas, dificultando a funcionalidade, o que parece ter melhorado após o protocolo.

Os dados baropodométricos, referentes à variação da superfície $\left(\mathrm{cm}^{2}\right)$, mostraram valor menor após intervenção. A variável velocidade média $(\mathrm{mm} / \mathrm{s})$ evidenciou aumento após atividade, os indivíduos tendem a andar mais rápido. Observou-se ainda que, na distância entre 2 ㅇ e 3 으 passo $(\mathrm{cm})$ e entre 3ㅇ e 4으 passo $(\mathrm{cm})$, os indivíduos com intervenção parecem tê-las aumentado, exceto esta última para o primeiro paciente. Distâncias percorridas em pequenos espaços propuseram tendência a uma maior velocidade da marcha e ao consequente aumento da segurança dos pacientes durante a deambulação, o que evidencia a possibilidade de melhora da marcha, em razão da autoconfiança e solidez das passadas.

Deficiências da marcha e quedas são frequentes em idosos e pacientes com doenças neurológicas. Aproximadamente $30 \%$ dos adultos acima de 65 anos caem pelo menos uma vez por ano. Já indivíduos com DP, em que há comprometimento cognitivo leve e demência, e, apesar do equilíbrio estar geralmente preservado na DP idiopática, as quedas aumentam para $60-80 \% .^{19}$ Com o protocolo de Nitendo Wii Fit, participantes relataram maior agilidade e segurança nas passadas, o que pode ser comprovado pelo aumento da velocidade média dessas.

A intervenção nestes casos é voltada para melhora na qualidade de vida e manutenção da funcionalidade. A RV é um recurso utilizado nessa linha terapêutica, pois permite prática de atividades em ambientes seguros e desafiantes, permitindo aprendizagem motora consequente da neuroplasticidade. ${ }^{6}$

Neste estudo, utilizou-se Nintendo Wii, com sensores que captam movimento do corpo do jogador e os reproduz na tela, permitindo interação vídeo game-jogador do corpo todo. ${ }^{17}$

Uma forma lúdica de desenvolver independência funcional nos indivíduos parkinsonianos, como ferramenta de busca de transferência de função para ambiente real no cotidiano desses pacientes, é a RV imersiva simulando ambiente domiciliar oferecendo abordagens neuropsicológicas e limites de superação inerentes. ${ }^{20}$

Dados semelhantes também se apresentaram sugestivos na presente pesquisa mediante achados não quantificados de passagem de fase, comparação entre os colegas de jogo e encanto com a ferramenta.

A variação de superfície direita e esquerda $\left(\mathrm{cm}^{2}\right)$ supõe aumento dos valores após 3 semanas de intervenção apenas no segundo paciente. Para que um movimento se torne automático, a eficiência neural é aumentada, sendo as informações armazenadas ou facilitadas no território sensorial do estriado.
Na patologia abordada, pela degeneração nigro-estriatal, o padrão automático está comprometido, podendo ser modulado pela atenção, ${ }^{21}$ parâmetro utilizado nesse protocolo para que os pacientes pudessem alcançar o objetivo dos jogos.

Oscilações ântero-posterior $(\mathrm{cm})$ do paciente 2, láterolateral esquerdo $(\mathrm{cm})$ do paciente 1 e ântero-posterior do corpo $(\mathrm{cm})$ evidenciam acréscimo na fase final de aplicação do protocolo. Essas circunstâncias podem estar relacionadas à melhora da oscilação pós intervenção, em que o aumento da oscilação sugere maior estabilidade e consequente controle de movimentos, já que, no Parkinson, os movimentos costumam ser limitados e a rigidez muscular predomina nos doentes. ${ }^{22,23}$

Outra evidência importante foi o aumento dos valores da variável distância entre o 20 e 30 passo $(\mathrm{cm})$, provavelmente pela maior distância percorrida da pisada, que requer menor velocidade para desenvolvê-la. Estudo demonstrou que o exercício melhora o desempenho motor e deve a isso diferentes aspectos da transmissão dopaminérgica e glutamatérgica. ${ }^{24}$

Decorridas 6 semanas de início do estudo estabelecido, a variável velocidade média $(\mathrm{mm} / \mathrm{s})$ apresentou valores aumentados ao final do protocolo, exceto do lado esquerdo do primeiro paciente.

A análise cinemática avalia comprimento da passada e cadência. A cadência relaciona-se à regulação do comprimento da passada. Um treinamento prolongado e que requer atenção pode gerar comprimento adequado da passada em pacientes acometidos, ${ }^{25}$ embora não evidenciado no presente estudo, talvez pela duração do protocolo.

Uma investigação em membros inferiores de sujeitos com DP verificou, dentre outras variáveis, taxa de desenvolvimento de força com estratégia de extensão isométrica do joelho. Ficou clara a associação direta entre ativação muscular agonista e taxa de desenvolvimento de força, com maior controle de movimento, ${ }^{26}$ corroborando, assim, o presente estudo, no qual os pacientes evidenciaram uma melhora no equilíbrio estático e na marcha.

Atividade física configura-se como benéfica para a prevenção de inúmeras patologias e riscos associados. ${ }^{15} \mathrm{O}$ estudo em questão tende a contribuir para a melhora da estabilidade ortostática e da deambulação nesses pacientes em virtude dos objetivos dos jogos utilizados nesse protocolo.

\section{CONCLUSÃO}

O uso de jogos em realidade virtual, por envolver dinamicidade e pontuação, mostra-se como um método inovador no tratamento motor da Doença de Parkinson, uma vez que os pacientes tornam-se mais incentivados mediante comparação entre os colegas.

Diante disso, os exercícios protocolados em pacientes com DP por meio da realidade virtual Nintendo $W_{i i}$ melhoraram o equilíbrio estático e a marcha, mediante ganho de desempenho motor e aptidão física, o que tende a favorecer a capacidade funcional e a deambulação, repercutindo, assim, positivamente, de maneira indireta, na qualidade de vida desses pacientes. Entretanto, sugere-se ainda a realização de mais estudos com jogos de RV, com o fito de ampliar as variáveis de avaliação e a amostra de pacientes. 


\section{REFERÊNCIAS}

1. Aguiar PMC, Severino P. Biomarcadores na doença de Parkinson: avaliação da expressão gênica no sangue periférico de pacientes com e sem mutações nos genes PARK2 e PARK8. Einstein. 2010; 8 (3): 291-7. Doi: https://doi.org/10.1590/s1679-45082010ao1674

2. Leandro LA. Fatores associados ao desempenho funcional de idosos portadores da Doença de Parkinson [Dissertação]. Curitiba: Universidade Federal do Paraná; 2011.

3. Queiroz FS. Autocuidado e qualidade de vida de idosas com Parkinson e disfunção miccional [Dissertação]. Salvador: Universidade Federal da Bahia; 2012.

4. Peternella FMN, Marcon SS. Descobrindo a Doença de Parkinson: impacto para o parkinsoniano e seu familiar. Rev Bras Enferm. 2009;62(1):1-4. Doi: https://doi.org/10.1590/S0034-71672009000100004

5. Almeida MHM, Castiglioni MC. Recursos tecnológicos: estratégia de promoção do autocuidado, atividades e participação para pessoas com doença de Parkinson. Rev Ter Ocup Univ São Paulo. 2007;18(3):152-7. Doi: https://doi.org/10.11606/issn.2238-6149.v18i3p152-157

6. Molina KI, Ricci NA, Moraes SA. Virtual reality using games for improving physical functioning in older adults: a systematic review. J Neuroeng Rehabil. 2014; 11(1):156. Doi: https://doi.org/10.1186/1743-0003-11-156

7. Fontoura VCB, Macêdo JGF, Silva LP, Silva IB, Coriolano MGWS, Monteiro D. Papel da reabilitação com realidade virtual na capacidade funcional e qualidade de vida de indivíduos com doença de Parkinson. Acta Fisiátr.2017;24(2):86-91. https://doi.org/10.5935/0104-7795.20170017

8. Barry G, Galna B, Rochester L. The role of exergaming in Parkinson's disease rehabilitation: a systematic review of the evidence. J Neuroeng Rehabil. 2014;11 (1):33. Doi: https://doi.org/10.1186/1743-0003-11-33

9. Goble DJ, Cone BL, Fling BW. Using the Wii Fit as a tool for balance assessment and neurorehabilitation: the first half decade of "Wii-search". J Neuroeng Rehabil. 2014;11(1):12. Doi: https://doi.org/10.1186/1743-0003-11-12

10. Hoehn MM, Yahr MD. Parkinsonism onset, progression, and mortality. Neurology. 1967;17(5): 427-42. Doi: https://doi.org/10.1212/wnl.17.5.427

11. Langston JW, Widner H, Goetz CG, Brooks D, Fahn S, Freeman $T$, et al. Core assessment program for intracerebral transplantations (CAPIT). Mov Disord. 1992;7(1):2-13.

https://doi.org/10.1002/mds.870070103

12. Deacon M, Parsons J, Mathieson S, Davies TC. Can Wii Balance? Evaluating a Stepping Game for Older Adults. IEEE Trans Neural Syst Rehabil Eng. 2018;26(9):1783-93. Doi: https://doi.org/10.1109/TNSRE.2018.2862146

13. Mengarelli A, Cardarelli S, Strazza A, Di Nardo F, Fioretti S, Verdini F. Validity of the Nintendo Wii balance board for the Assessment of Balance Measures in the Functional Reach Test. IEEE Trans Neural Syst Rehabil Eng. 2018;26(7):14001406. Doi: https://doi.org/10.1109/TNSRE.2018.2843884
14. Araújo LP, Rodrigues AP. Exercício físico e avaliação da capacidade funcional de idosos com Parkinson. Motricidade. 2018;14(SI):54-9. Doi: https://doi.org/10.6063/motricidade.16237

15. Crocetta TB, Oliveira SR, Liz CM, Andrade A. Tecnologias de realidade virtual e aumentada no desempenho humano: uma revisão. Fisioter Mov. 2015;28(4):823-35. Doi: https://doi.org/10.1590/0103-5150.028.004.AR01

16. Xu Q, Park Y, Huang X, Hollenbeck A, Blair A, Schatzkin A, et al. Physical activities and future risk of Parkinson disease. Neurology. 2010;75(4):341-8. Doi: https://doi.org/10.1212/WNL.0b013e3181ea1597

17. Paula FR, Lima LO, Salmela LFT. Exercício aeróbio e fortalecimento muscular melhoram o desempenho funcional na doença de Parkinson. Fisioter Mov. 2011; 24(3):379-88. Doi: https://doi.org/10.1590/S010351502011000300002

18. Fonseca LJP, Brandalize M, Brandalize D. Nintendo Wii na reabilitação de pacientes com paralisia cerebral - relato de caso. Arq Ciênc Saúde UNIPAR. 2012;16(1): 39-43. Doi: https://doi.org/10.25110/arqsaude.v16i1.2012.4565

19. Descatoire A, Thévenon A, Moretto A. Baropodometric information return device for foot unloading. Med Eng Phys. 2009;31(5):607-13. Doi: https://doi.org/10.1016/j.medengphy.2008.12.002

20. Klinger E, Chemin I, Lebreton S, Marié RM. Virtual action planning in Parkinson's disease: a control study. Cyberpsychol Behav. 2006;9(3):342-7.Doi: https://doi.org/10.1089/cpb.2006.9.342

21. Ebersbach G, Grust U, Ebersbach A, Wegner B, Gandor F, Kühn AA. Amplitude-oriented exercise in Parkinson's disease: a randomized study comparing LSVT-BIG and a short training protocol. J Neural Transm. 2015;122(2): 2536. Doi: https://doi.org/10.1007/s00702-014-1245-8

22. Fukunaga JY, Quitschal RM, Doná F, Ferraz HB, Ganança $\mathrm{MM}$, Caovilla HH. Postural control in Parkinson's disease. Braz J Otorhinolaryngol. 2014;80(6):508-514. Doi: https://doi.org/10.1016/j.bjorl.2014.05.032

23. Sánchez-Arias MDR, Kleiner AFR, Hamanaka AYY, Santiago PRP, Gobbi LTB, Stella F. Restrição visual e oscilações corporais ântero-posteriores na doença de Parkinson. Acta Fisiátr. 2012;19(3):161-6. Doi: https://doi.org/10.5935/0104-7795.20120025

24. Frazzitta G, Maestri R, Ghilardi MF, Riboldazzi G, Perini M, Bertotti $G$, et al. Intensive rehabilitation increases BDNF serum levels in parkinsonian patients: a randomized study. Neurorehabil Neural Repair. 2014 Feb;28(2):163-8. Doi: https://doi.org/10.1177/1545968313508474

25. Gonçalves GB, Leite MA, Pereira JS. Influência das distintas modalidade de reabilitação sobre as disfunções motoras decorrentes da Doença de Parkinson. Rev Bras Neurol. 2011; 47 (2): 22-30.

26. Rose MH, Lokkegaard A, Sonne-holm S. Tremor irregularity, torque steadiness and rate of force development in Parkinson's disease. Motor Control. 2012; 17(2):203-16. Doi: https://doi.org/10.1123/mcj.17.2.20 